\title{
Demo Abstract: An Intuitive Drag and Drop Framework for Wireless Network Experimentation
}

\author{
Michael Tetemke Mehari*, Adnan Shahid, Ingrid Moerman, Eli De Poorter \\ Ghent University - imec, IDLab, Department of Information Technology \\ Ghent, Belgium \\ \{michael.mehari,adnan.shahid,ingrid.moerman,eli.depoorter\}@ugent.be
}

\begin{abstract}
Experimental wireless network research is often very time consuming and requires knowledge of multiple experimentation platforms (JFED, OMF, etc.), thereby hindering innovation specially from non-testbed experts. To foster innovation, this paper presents an intuitive wireless experimentation using the Node-RED framework. Within the framework, drag and drop components are combined to set-up wireless experiments in simulation and testbed environments, configure network stack and execute series of experiments. Furthermore, the intuitiveness of the Node-RED framework is demonstrated by using drag and drop components to optimize multiple conflicting objectives in simulation and in a real-testbed, without requiring advanced testbed knowledge.
\end{abstract}

\section{CCS CONCEPTS}

- Networks $\rightarrow$ Network experimentation;

\section{KEYWORDS}

wireless network experimentation, optimization, Node-RED, dragand-drop experimentation, wireless testbed facilities

\section{ACM Reference Format:}

Michael Tetemke Mehari*, Adnan Shahid, Ingrid Moerman, Eli De Poorter. 2017. Demo Abstract: An Intuitive Drag and Drop Framework for Wireless Network Experimentation. In Proceedings of SenSys '17. ACM, New York, NY, USA, 2 pages. https://doi.org/10.1145/3131672.3136971

\section{INTRODUCTION}

The demand for wireless networks that offer advanced services requires increasingly complex innovations from the research community. However, to cope with the needs of end users, researchers need to mock-up the wireless system by using simulations or experimental testbeds. In contrast to simulators, using wireless testbeds has a steep learning curve, mainly due to complex management and orchestration frameworks, software libraries and storage databases. For example, to measure the maximum throughput of a Wi-Fi network on a wireless testbed, we first need to reserve $N$ wireless nodes and flash them with an operating system. Next the user connects

Permission to make digital or hard copies of all or part of this work for personal or classroom use is granted without fee provided that copies are not made or distributed for profit or commercial advantage and that copies bear this notice and the full citation on the first page. Copyrights for components of this work owned by others than the author(s) must be honored. Abstracting with credit is permitted. To copy otherwise, or republish, to post on servers or to redistribute to lists, requires prior specific permission and/or a fee. Request permissions from permissions@acm.org.

SenSys '17, November 6-8, 2017, Delft, Netherlands

(c) 2017 Copyright held by the owner/author(s). Publication rights licensed to Association for Computing Machinery.

ACM ISBN 978-1-4503-5459-2/17/11 \$\$15.00

https://doi.org/10.1145/3131672.3136971 to each node, creates a Wi-Fi connection, starts an iperf instance and measures the network throughput. This manual approach is tedious and gets increasingly complex when the network grows. The required effort can be reduced by using an orchestration framework, which hides the distributed manual scripting by a centralized automated system. Afterwards, results are stored in a central database, yet requiring another technology. As such, a researcher who wants to validate wireless problems will encounter a steep learning curve even before doing the simplest experiment, since many of the tools differ between testbeds and/or simulators.

In this demo paper, a graphical wireless experimentation is demonstrated as opposed to the traditional experimentation. To this end, the Node-RED [3] framework is used which offers a flow based drag and drop experimentation. By using reusable components and applying drag and drop operation, the Node-RED framework lowers the experimentation effort which otherwise is required and allow more time to be spent on the core wireless problem.

\section{DEMONSTRATION}

\subsection{Drag and Drop Components}

Experiments can be created by combining drag-and-drop components from multiple categories, which are listed in Table 1.

Table 1: Node-RED framework drag and drop components

\begin{tabular}{|l|l|}
\hline Category & Components \\
\hline Environment & Simulation (cooja), Testbed (WISHFUL portable, w-lab.t) \\
\hline Configuration & TAISC, Contiki, Wireless MAC Processor (WMP) \\
\hline Planning & Exhaustive, Random, Locating array, Latin Hypercube Designer (LHD) \\
\hline Metrics & packet loss, RSSI, LQI, latency, energy consumption \\
\hline Storage & file, database, REST API \\
\hline Reasoning & Optimization (surrogate), Model Creation (kriging), Sensitivity Analysis \\
\hline
\end{tabular}

The first category of components reserves and initializes the correct environment, allowing to define which simulator (e.g Cooja) or testbed facility (e.g. WISHFUL portable testbed [5] or w-iLab.t wireless testbed [1]) should be used. Each environment supports several software platforms (Contiki, TAISC, WMP) with multiple configurable parameters. Each of these platforms is also represented as a separate drag-and-drop component, which can be double-clicked for intuitive configuration. When doing multiple experiments, the planning components define how the parameters should be changed between each experiment iteration, for example for exploration purposes (e.g. latin hypercube sampling) or for model creation (e.g. exhaustive searching). It is also possible to define which performance metrics need to be calculated and how they should be stored. Finally, several pre-defined reasoning components are available to process the collected metrics, e.g. to find optimum settings, to 
create system models or to analyze the sensitivity of parameters. Node-RED also allows to create and share new components, making the framework easily extensible by external users.

\subsection{Scenario}

To demonstrate the intuitiveness of the Node-RED framework, a single-hop Wireless Sensor Network (WSN) is optimized using a SUrrogate MOdelling (SUMO) toolbox [2]. The WSN consists of 30 source nodes sending periodic reports to a sink node. During each experiment iteration, the source nodes update four parameters (txPower at PHY layer and cycletime, CCA check count, retransmission count at contiki-MAC layer) and two performance metrics (i.e. Energy consumption and Latency) are calculated afterwards. Each configurable parameter affects the objectives differently. Increasing cycletime, for example, favors energy consumption but at the expense of increased latency. To a lesser extent, decreasing txPower also favors energy consumption but affects latency. CCA check count and retransmission count, on the other hand, negatively affect energy consumption and latency objectives respectively.

\subsection{Node-RED Flow}

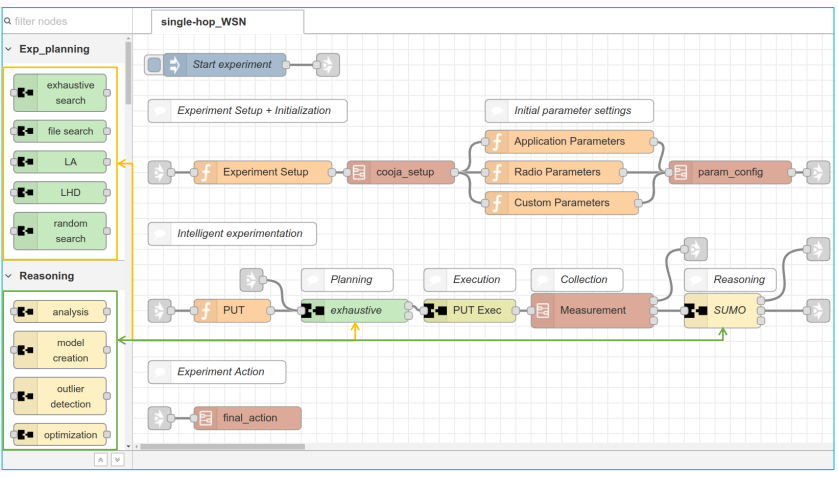

Figure 1: Node-RED experimentation flow. Left side column categorizes components and right side plane builds an experiment flow by dragging and dropping available components.

A Node-RED flow for the single-hop WSN scenario is shown in Figure 1. All orange nodes (i.e. Node-RED function nodes) are used to describe the experiment whereas the remaining nodes are involved in the actual experimentation. Components marked in a darker red color represent sub-flows that can be opened in a new tab to see and/or modify the underlying flow. Beginning from the top left corner, an experiment is started by clicking the start button and it triggers standardized JSON messages to pass through the nodes. First the experiment is setup and initialized using default parameters. Next parameters are defined inside the PUT function node. Afterwards, the experiment enters a cognitive loop where planing, configuration, sensing and reasoning blocks dictate the experiment. If the reasoning block succeeds meeting a stopping criteria, a final action is performed else the cognitive loop continues operation. In our example, the scenario is optimized using LHD planner and SUMO optimizer and the result is shown in Figure 2.

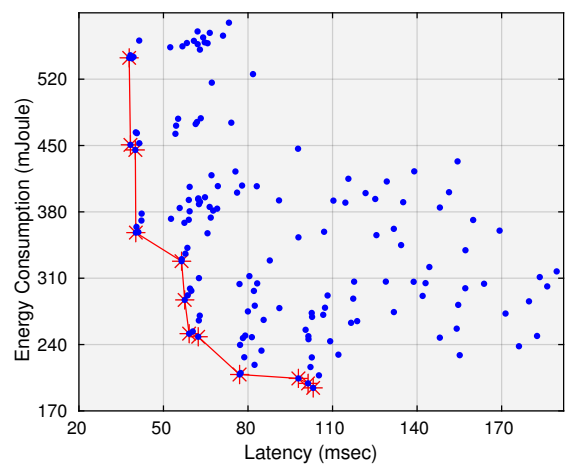

Figure 2: SUMO performance plot. Reducing energy consumption and latency being the objective, the Pareto front (red line) shows the optimum performance dominating remaining points (blue dots).

\subsection{Node-RED Customization}

Researchers can repeat the same experiment setup and validate the scenario at different moments in time using different testbed facilities. During the demonstration, researchers can play with the drag-and-drop framework to create their own variations of the experiment. For example, it makes sense to simulate the experiment first and then move on to a real testbed once results are satisfactory. This can be easily done by using a WISHFUL portable setup node from the repository. Or we might want to investigate different parameters by modifying the PUT function node. Similarly, the experiment can also be customized by using various components from the planning and reasoning repositories.

\section{CONCLUSION}

This demo paper presented an intuitive drag and drop approach for wireless experimentation. To this end, a Node-RED framework is used that offers a unique advantage by hiding experimentation details and leaving room for the core wireless problem. Moreover, experiment customization is simplified by using drag and drop components which are easily shared among research peers. Finally, a single-hop WSN is optimized demonstrating the intuitiveness of the framework. The code used in this paper is available on [4].

\section{ACKNOWLEDGMENTS}

The research leading to these results has received funding from the European Horizon 2020 Program (under grant agreement No. 688116 eWINE Project [https://ewine-project.eu]) and the FWOSBO SAMURAI project [http://www.samurai-project.be].

\section{REFERENCES}

[1] Stefan Bouckaert, Pieter Becue, Brecht Vermeulen, Bart Jooris, Ingrid Moerman, and Piet Demeester. 2012. Federating Wired and Wireless Test Facilities through Emulab and OMF: The iLab.t Use Case. Vol. 44. 305-320.

[2] Dirk Gorissen, Ivo Couckuyt, Piet Demeester, Tom Dhaene, and Karel Crombecq. 2010. A surrogate modeling and adaptive sampling toolbox for computer based design. FOURNAL OF MACHINE LEARNING RESEARCH (2010).

[3] IBM. 2017. Flow-based programming for the IoT. (08 2017). https://nodered.org

[4] Michael Tetemke Mehari. 2017. Node-RED framework GitHub repository. (Aug 2017). https://github.com/mmehari/Node-RED_Framework

[5] Ingrid Moerman. 2016. WISHFUL Portable testbed. (Feb 2016). http://www. wishful-project.eu/node/16 